\title{
Synthesis and characterization of hybrid nanostructures
}

\section{Taleb Mokari*}

\author{
Department of Chemistry and Ilse Katz Institute for Nanoscale Science and Technology, Ben-Gurion \\ University of the Negev, Beer Sheva, Israel
}

Received: 19 January 2011; Revised: 24 March 2011; Accepted: 3 April 2011; Published: 4 May 2011

\section{Abstract}

There has been significant interest in the development of multicomponent nanocrystals formed by the assembly of two or more different materials with control over size, shape, composition, and spatial orientation. In particular, the selective growth of metals on the tips of semiconductor nanorods and wires can act to couple the electrical and optical properties of semiconductors with the unique properties of various metals. Here, we outline our progress on the solution-phase synthesis of metal-semiconductor heterojunctions formed by the growth of $\mathrm{Au}, \mathrm{Pt}$, or other binary catalytic metal systems on metal $(\mathrm{Cd}, \mathrm{Pb}, \mathrm{Cu})$-chalcogenide nanostructures. We show the ability to grow the metal on various shapes (spherical, rods, hexagonal prisms, and wires). Furthermore, manipulating the composition of the metal nanoparticles is also shown, where $\mathrm{PtNi}$ and $\mathrm{PtCo}$ alloys are our main focus. The magnetic and electrical properties of the developed hybrid nanostructures are shown.

Keywords: synthesis; Hybrid Nanocrystals; Nanowires; Electrical and magnetic properties; MOCVD he assembly of two or more nanostructured components with precise control over the size, shape, composition, and spatial orientation is desirable not only for the combination of different functionalities, but also for advanced properties that can arise independent of the single-component materials. Engineering hybrid multicomponent nanostructures draws on the vast array of synthetic techniques now at our disposal to assemble nanocrystals with very different properties; a semiconductor nanocrystal can be combined with a metal in such a way that the hybrid structure can be tailored to a specific application.

Non-centrosymmetric hybrid nanocrystals have been prepared from a variety of materials combinations, with the resulting conformations depending on the crystal structure, miscibility, interfacial strain, or selective

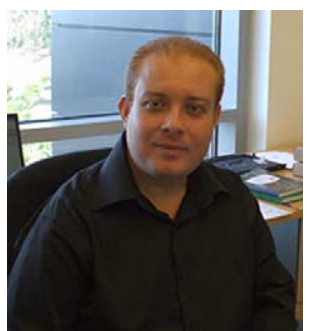

Taleb Mokari received a BSc in chemistry from the Hebrew University in 2000, an MSc. and a PhD from Hebrew University in 2002 and 2006. He was a Fulbright and Ilan Ramon postdoctoral fellow at the University of California-Berkeley for one year. He joined the Molecular Foundry at LBNL as a staff scientist in 2007. He has been a faculty member at the Department of Chemistry, Ben-Gurion University since December 2009. reactivity of the components (1). In this type of hybrid nanocrystal, both materials are exposed and spatially isolated, allowing for asymmetric assembly or functionalization. This is, in contrast to core/shell structures, where only the outer shell is available for chemical reactions. Semiconductors, for example, often exhibit considerably greater facet-specific chemical reactivity than metals, owing to their anisotropic crystal structures (2). There has been significant interest in the selective growth of metals on the tips of nanorods and wires to couple the electrical and optical properties of semiconductors with the unique properties of various metals. A number of approaches have been used to selectively grow metals on semiconductors through reduction, physical deposition, or photochemistry to give $\mathrm{Au}$ and $\mathrm{Ag}$ on $\mathrm{ZnO}(3,4)$, Co and $\mathrm{Au}$ on $\mathrm{TiO}_{2}(5,6)$, and others $(7,8)$. Here we describe 
our progress on the development of metal-semiconductor heterojunctions focused on $\mathrm{Cd}$ and $\mathrm{Pb}$ calcogenide interfaces with $\mathrm{Au}, \mathrm{Pt}$, and other binary catalytic metal systems.

A number of bimetallic nanoparticle systems, including the $\mathrm{Ni}_{\mathrm{x}} \mathrm{Pt}_{1-\mathrm{x}}$ nanocrystals described by Weller and coworkers, have magnetic properties that can be utilized for high-density data storage and other magnetic-based applications (9). Furthermore, bimetallic materials frequently present advanced catalytic behaviors distinct from their constituent metals. For example, NiPt nanoparticles show enhanced activity for oxygen reduction relative to pure $\mathrm{Pt}(10,11)$. The incorporation of semiconductors with bimetallic catalyst particles may be of particular interest for photocatalytic and photovoltaic applications.

\section{Preparation of hybrid nanostructures}

\section{Colse nanowires}

CdSe nanowires were grown by a previously reported form (12) of the SLS growth mechanism (13). Briefly, cadmium oxide (CdO 99.99\%, $0.006 \mathrm{~g}, 0.047 \mathrm{mmol}$ ) was complexed with $n$-tetradecylphosphonic acid (n-TDPA $97 \%, 0.0270 \mathrm{~g}, 0.097 \mathrm{mmol})$ in trioctylphosphine oxide (TOPO $99 \%, 5 \mathrm{gr}$ ) for $90 \mathrm{~min}$ at $300^{\circ} \mathrm{C}$ under $\mathrm{N}_{2}$ using standard Schlenk technique. The catalyst, a $6 \mathrm{~nm}$ thin film of $\mathrm{Bi}$, was thermally evaporated on top of a $2.5 \mathrm{~nm}$ sticking layer of $\mathrm{Cr}$ on a $\mathrm{Si}(100)$ substrate. In a typical synthesis, one or two $\sim 0.4 \mathrm{~cm}^{2} \mathrm{Si}$ substrates were added to the Cd-TDPA solution at $270^{\circ} \mathrm{C}$. Since the bulk melting point of $\mathrm{Bi}$ is $271^{\circ} \mathrm{C}$, some of the $\mathrm{Bi}$ thin film melts and dewets from the surface of the substrate forming Bi nanoparticle catalysts. Next, $0.25 \mathrm{~mL}$ of 2.5 $\mathrm{wt}^{\mathrm{t}} \%$ selenium $(99.5 \%$ powder mesh) dissolved in trioctylphosphine $(97 \%)$ was injected into the solution. After 5 min of growth time, the solution was cooled and then diluted with $5-10 \mathrm{~mL}$ of toluene. The CdSe nanowires suspended in solution were precipitated by addition of methanol followed by centrifugation. The product was stored as a colloidal suspension in toluene.

\section{$\mathrm{Cu}_{2} \mathrm{~S}$ nanoprisms}

In a typical synthesis of the hexagonal nanoprisms, $6 \mathrm{~mL}$ of a $0.11 \mathrm{M}$ solution of $\mathrm{Cu}(\mathrm{II})\left[\mathrm{S}_{2} \mathrm{CNC}_{4} \mathrm{H}_{10}\right]_{2}$ in trioctylphosphine (TOP 97\%) is placed on a $6 \mathrm{~mm}-6 \mathrm{~mm} \mathrm{Si}(100)$ substrate that has been cleaned by sonication in isopropanol. The substrate is placed onto a glass slide on a hot plate and heated under inert atmosphere (N2) to $240-250^{\circ} \mathrm{C}$ until all solvent has evaporated (about $10 \mathrm{~min}$ ).

Single-source molecular precursors $\mathrm{Cu}(\mathrm{II})\left[\mathrm{S}_{2} \mathrm{CNC}_{4}\right.$ $\left.\mathrm{H}_{10}\right]_{2}$ and was synthesized based on previously published methods (14). Briefly, $\mathrm{Cu}(\mathrm{II}) \mathrm{Cl}_{2}, \mathrm{~Pb}(\mathrm{II}) \mathrm{Cl}_{2}$, and sodium diethyldithiocarbamate $\left(\mathrm{Na}(\mathrm{I})\left[\mathrm{S}_{2} \mathrm{CNC}_{4} \mathrm{H}_{10}\right]\right)$ were dissolved separately in deionized water $(\mathrm{R}>18.0 \mathrm{MU})$. The metal ion solution was mixed with the dissolved diethyldithiocarbamate ligand solution with a ligand to metal molar ratio of $2: 1$. The product formed immediately as a precipitate and was vacuum filtered and dried. The molecular precursor was recrystallized once from hot chloroform before use.

\section{Au growth}

The CdSe wires were decorated with $\mathrm{Au}$ according to a method described previously (15). A toluene solution containing gold trichloride $\left(\mathrm{AuCl}_{3}, 12 \mathrm{mg}, 0.03 \mathrm{mmol}\right)$, didodecyldimethylammonium bromide (DDAB, $40 \mathrm{mg}$, $0.09 \mathrm{mmol}$ ), and dodecylamine (DDA, $70 \mathrm{mg}, 0.38$ mmol) was sonicated for $5 \mathrm{~min}$ and then added dropwise to a suspension of nanowires stirring under $\mathrm{N}_{2}$. The product was precipitated with methanol and separated by centrifugation. The amount of precursor added was varied to give the desired degree of Au overgrowth.

\section{Pt, PtNi, and PtCo growth}

Decoration of the CdSe wires with $\mathrm{Pt}, \mathrm{PtNi}$, and $\mathrm{PtCo}$ was achieved as described previously (16). Oleic acid $(0.2$ $\mathrm{mL})$, oleylamine $(0.2 \mathrm{~mL})$, and 1, 2-hexadecanediol (43.0 $\mathrm{mg})$ were heated in diphenyl ether $(10 \mathrm{~mL})$ at $80^{\circ} \mathrm{C}$ under vacuum for $30 \mathrm{~min}$ to remove traces of water. Platinum acetylacetonate $(32.8 \mathrm{mg})$ was added to a suspension of $\mathrm{CdSe}$ nanowires in 1,2-dichlorobenzene and heated at $65^{\circ} \mathrm{C}$ for $10 \mathrm{~min}$ to promote dissolution of the $\mathrm{Pt}$ precursor. The mixture of surfactants and diphenyl ether was purged with nitrogen and heated to $200^{\circ} \mathrm{C}$ before injecting the Pt precursor and semiconductor wires. After several minutes, the reaction was removed from heat and quenched in a water bath. The product was washed by precipitation with ethanol followed by centrifugation. Either nickel or cobalt acetate was added to the reaction prior to heating to $80^{\circ} \mathrm{C}$ to promote $\mathrm{PtNi}$ or $\mathrm{PtCo}$ formation. Control over the binary composition is possible by altering the ratio of the metallic components or by changing the metal to $\mathrm{CdSe}$ nanowire ratio.

\section{GaN and InGaN growth}

GaN and GaN@InGaN core-shell nanowire growth was carried out in a Thomas Swan, Showerhead MOCVD cold-wall reactor. The growth substrates were prepared by drop-casting $40 \mu \mathrm{L}$ of $0.2 \mathrm{M}$ solution of $\mathrm{NiNO}_{3}$ in ethanol onto $1 \mathrm{~cm}^{2}$ c-plane sapphire wafer. For substrates, of 5:1 ratio B. B. International $10 \mathrm{~nm}$ gold colloid to ethanol was drop-cast onto wafers. Pure GaN nanowires were grown for $10 \mathrm{~min}$ at $\sim 825^{\circ} \mathrm{C}$. The reactive and carrier gas flows were $600,41,200$, and $400 \mathrm{sccm}$ for ammonia, tri-methyl gallium (TMG), hydrogen, and nitrogen. The TMG bubbler was kept at $5^{\circ} \mathrm{C}$ and the reactor pressure was 140 Torr. For core-shell wires, the same conditions were used for $\mathrm{GaN}$ nanowires with a subsequent step for InGaN deposition. The InGaN shells 
were grown for 15 min with temperatures from 700 to $750^{\circ} \mathrm{C}$. The reactive and carrier gas flows were 5,000 , 1,190 , and $1,000 \mathrm{sccm}$ for ammonia, TMG, tri-methyl Indium (TMI), and nitrogen. The TMI bubbler was kept at $18^{\circ} \mathrm{C}$ and the reactor pressure was 400 Torr.

\section{Characterization}

The structure and composition of the samples were investigated by TEM and HRTEM (Tecnai G2 S-Twin microscope operated at $200 \mathrm{kV}$ and JEOL 2100-F FieldEmission Analytical Transmission Electron Microscope operated at $200 \mathrm{kV}$ ), EDS (Philips CM200/FEG microscope operated at $200 \mathrm{kV}$, equipped with a Link EDS detector, and a Weiss FESEM Ultra-55 operated at 30 $\mathrm{kV}$ ), and XRD (Bruker-AXS D8 with a general area detector and Co $\mathrm{K} \alpha$ radiation). Samples were dispersed on carbon coated copper grids for TEM or glass substrates for XRD. Toluene suspensions of the nanowires were prepared for analysis by UV-vis absorption spectroscopy (Shimadzu UV-3,600), and PL spectroscopy was performed using a $532 \mathrm{~nm}$ excitation source for disperse samples on TEM grids. Image correlation for PL measurements was performed using the STEM mode on a Weiss FESEM Ultra-55 operating at $30 \mathrm{kV}$. Magnetic measurements were performed on powder samples of the PtCo-overgrown CdSe nanowires with a SQUID magnetometer (Quantum Design MPMS XL).

\section{Conductance measurements}

Source and drain electrodes with junctions spanning 20$40 \mathrm{~nm}$ were fabricated on silicon wafer test chips using ebeam lithography, followed by deposition of $55 \mathrm{~nm}$ of $\mathrm{Au}$ on $10 \mathrm{~nm}$ of Ti. A film of $10 \mathrm{~nm}$ of silicon nitride or aluminum oxide was deposited via atomic layer deposition (ALD) before the electrodes to prevent shorting through the silicon substrate.

The nanorod samples were adsorbed to these predefined $\mathrm{Au}$ junctions by submerging the chip in the appropriate $\mu \mathrm{M}$ nanorod-toluene solution and drying with $\mathrm{N}_{2}$. The details of this process are discussed below. Once fabricated, the current through individual nanorod devices was characterized in a two-terminal geometry as a function of source-drain voltage and temperature. All data reported here were measured in a $10^{-6}$ Torr atmosphere with a Janis Research ST-500-2 micromanipulated probe station, chilled with liquid $\mathrm{He}$ or $\mathrm{N}_{2}$ cryogen.

\section{Properties of hybrid nanostructures}

There has been considerable interest in the selective growth of metals on semiconductors through reduction, physical deposition, or photochemistry. A facile method for the deposition of $\mathrm{Au}$ on the tips of $\mathrm{CdSe}$ nanorods was developed by one of the co-authors of this paper, based on the reduction of $\mathrm{Au}$ by dodecylamine in the presence of didodecyldimethylammonium bromide
(DDAB) at room temperature (15). Fig. 1A presents a TEM image of CdSe nanorods decorated with two gold tips. The same process has been shown for CdS nanorods (Fig. 1B) (17). The anisotropic wurtzite crystal structure of CdSe nanorods promotes selective growth of metals at the tips as a result of their higher reactivity that is also responsible for longitudinal growth along the $<001>$ direction to form CdSe nanorods. Initially, when the concentration of $\mathrm{Au}$ is low, growth dominates at one of the tips, possibly due to increased interaction with the Se rich facet. Along the $<001>$ axis the nanorods are alternately composed of planes of $\mathrm{Se}$ and $\mathrm{Cd}$, the $\mathrm{Cd}$ having less reactivity with the Au. As the reaction progresses and the $\mathrm{Au}$ concentration increases, the DDAB facilitates an etching process of the CdSe rods that may promote growth at both ends as Se sites are exposed on the other end. However, by increasing the ratio of $\mathrm{Au}$ to $\mathrm{CdSe}$ nanorods beyond the concentration needed for growth of two tips, another process occurs. In this process, one of the tips dissolves and the other tip grows, resulting in asymmetric heterostructures with single $\mathrm{Au}$ tips. This process was explained by an electron-transfer mediated ripening mechanism (18). These methods have also allowed for the selective growth of Au tips on CdSe rods (Fig. 1A) and CdTe tetrapods (Fig. 1C). We have shown that a variety of metals can be grown with high selectivity on the tips of Cd-calcogenides. In order to increase the application for metal-semiconductor heterostructures, the semiconductor can also be changed to include narrow band-gap $\mathrm{Pb}$ and $\mathrm{Cu}$-chalcogenide nanocrystals, for example. Metal growth has been demonstrated previously for $\mathrm{Au}$ on $\mathrm{PbS}$ (7), and we have shown that similar results occur for PbSe (Fig. 1E) and $\mathrm{Cu}_{2} \mathrm{~S}$ nanocrystals (Fig. 1D). The PbSe nanocrystals shown in Fig. 1E were treated with $\mathrm{AuCl}_{3}$ in toluene with dodecylamine and DDAB, and at early stages of growth or low $\mathrm{Au}$ concentrations the $\mathrm{PbSe}$ particles were decorated with multiple $\mathrm{Au}$ nucleates (Fig. 1E). At higher Au concentrations or a larger $\mathrm{Au}$ to $\mathrm{PbSe}$ ratio, a ripening process, similar to that observed for CdSe nanorods (18), resulted in coalescence of the $\mathrm{Au}$ nucleates on the PbSe nanocrystals. Growth of $\mathrm{Au}$ was expanded to gas phase (MOCVD) grown nanowires $(\mathrm{GaN}$ and $\mathrm{InGaN})$ and similar results were obtained as shown in Fig. 1F (19).

Extension of these methods to include Pt and related binary metals is of particular interest for photocatalytic applications where the catalytic activity of $\mathrm{Pt}$ is relevant for an extremely large number of reactions that are not accessible with Au. However, previous attempts to reduce $\mathrm{Pt}$ on CdSe in this manner were unsuccessful, and the reduction of $\mathrm{Au}$ on $\mathrm{CdS}$ resulted in the growth of metal clusters over the entire rod, with less selectivity for the tips (17). A high-temperature organometallic method for the synthesis of Pt-CdS, PtNi-CdS, and PtCo-CdS hybrid 

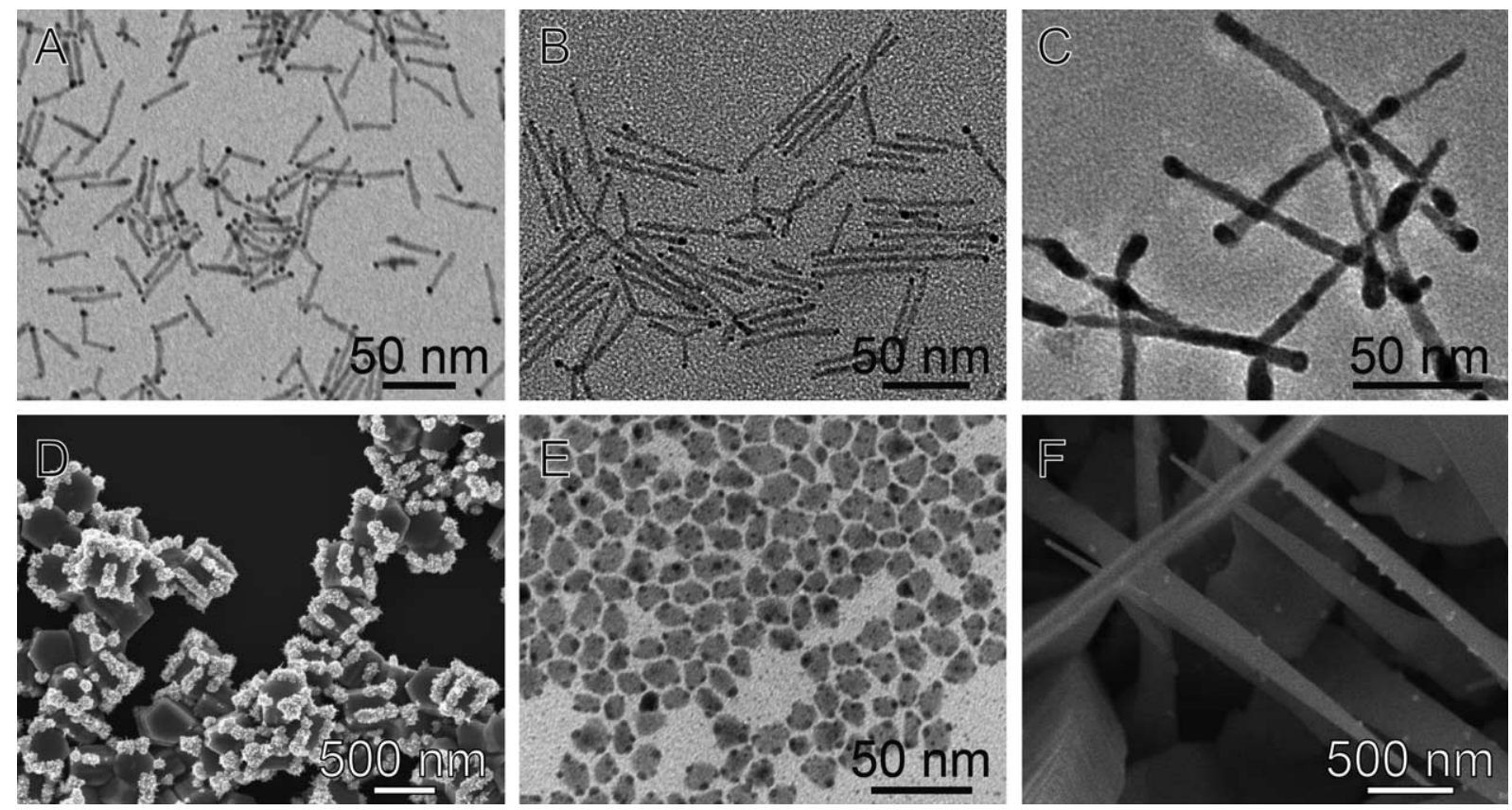

Fig. 1. Au overgrowth on semiconductor nanomaterials. (A) CdSe-Au nanorods, (B) CdS-Au nanorods, (C) CdTe-Au nanorods, (D) $\mathrm{Cu}_{2} \mathrm{~S}-\mathrm{Au}$ hexagonal nanoprisms, (E) PbSe-Au nanocrystals, (F) GaN-Au nanowires.

nanostructures with controllable size and composition is described here. There are several advantages inherent in this approach including high selectivity for the tips, an absence of etching that preserves the size of the nanorods, and composition control of the metal tips.

The procedure for the selective growth of $\mathrm{Pt}$ on semiconductor nanorods was adapted from a scheme for the synthesis of $\mathrm{Ni}_{\mathrm{x}} \mathrm{Pt}_{1-\mathrm{x}}$ nanoparticles developed by Weller and coworkers (9). In order to produce the heterostructures, a mixture of $\mathrm{Pt}$ precursor and $\mathrm{CdS}$ rods was injected into a coordinating solvent containing stabilizers and a reducing agent at high temperature, the growth of the Pt nanoparticles on CdS nanostructures was first shown in our earlier report (16). Herein, we discuss the growth of the four various compositions of metals on CdSe nanowires (20). We have used the same procedure that was described before to decorate CdSe nanowires with $\mathrm{Au}, \mathrm{Pt}$, and bimetal nanoparticles $(\mathrm{PtNi}$, $\mathrm{PtCo}$ ) as shown Fig. 2. Fig. 2A shows the Au growth on the surface of the CdSe nanowires. The size of the $\mathrm{Au}$ particles is controlled by changing the concentration of the Au precursor. The high reactivity of the tips as a result of increased surface energy and imperfect ligand passivation allows for selective chemistry on different facets (2). During the early stages of metal growth, or at a low metal concentration, metal deposition appears to occur preferentially on only one tip of the CdSe nanowires, this observation was clearly shown in the growth of the metal on the CdS (or CdSe) nanorods. This process is not related to the electrochemical ripening seen for the Au tipped semiconductors but rather can be explained by considering that at low metal concentrations, the Se rich facets along the $<001>$ axis are more highly reactive. Alternatively, due to the significant length of the CdSe nanowires relative to the size of the metal particles, each tip may act as an isolated nucleation site independent of the other tip. Thus, at low metal precursor concentrations where some of the rods do not experience metal growth on either tip, the remainder of metal nucleation will most likely occur on only one of the tips. Increasing the amount of metal relative to the concentration of $\mathrm{CdSe}$ wires leads to decoration of the nanowires with $\mathrm{Au}$ particles, as shown in Fig. 2A. The procedure was expanded to other metal as shown in Fig. 2B-D, which presents TEM images of CdSe nanowires decorated with $\mathrm{Pt}, \mathrm{PtNi}$, and $\mathrm{PtCo}$, respectively.

The XRD patterns and the EDS spectra of the four samples shown in Fig. 2 are presented in Fig. 3A, B. The pure CdSe wires and Pt-CdSe agree well with the bulk CdSe wurtzite crystal structure and that for face-centered cubic Pt. The EDS measurements shown in Fig. 3B of the CdSe and Pt-CdSe clearly show the elemental composition of the two samples $(\mathrm{Cd}, \mathrm{Se}$, and $\mathrm{Pt})$.

Characterization of the binary metallic heterostructures was performed to determine the composition of the metal tips. The XRD measurements (Fig. 3A) provide evidence for the presence of crystalline CdSe and Pt peak shifted corresponding to a change in lattice constant with alloy formation $(9,21)$. Furthermore, there was no evidence for pure $\mathrm{Ni}$ or $\mathrm{Co}$ phases or their respective 

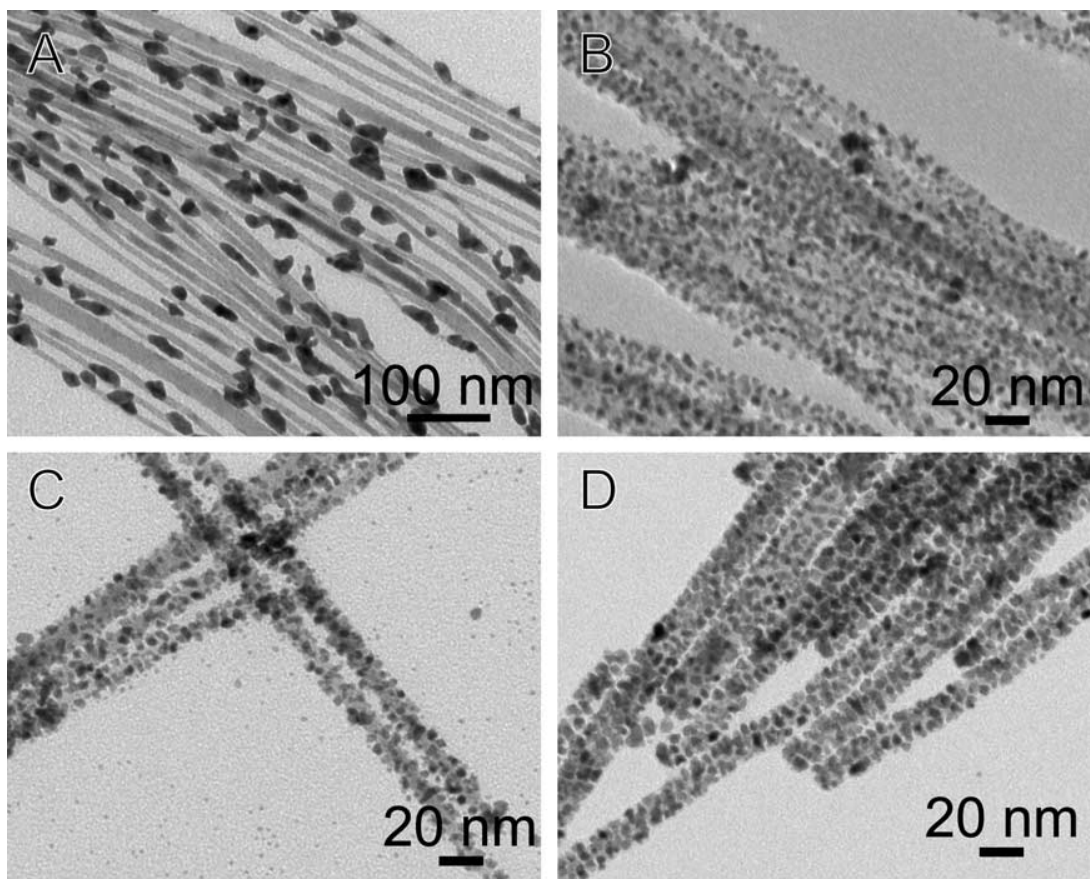

Fig. 2. Growth of different metals and binary metals on CdSe nanowires. (A) CdSe-Au nanowires, (B) CdSe-Pt nanowires, (C) CdSe-PtNi nanowires, (D) CdSe-PtCo nanowires.

oxides. Experimentally we have seen that the Co precursor is not reduced in the absence of Pt under the conditions described here. Selected area EDS measurements on a single tip of the PtNi-CdS hybrids shows the presence of both Pt and $\mathrm{Ni}$ in the tip (Fig. 3B), while analysis on a larger number of tips indicates the presence of approximately $25 \% \mathrm{Ni}$ relative to Pt. These results are of interest because control over the composition of the tips may have a direct effect on the catalytic activity of these materials.

Magnetic characterization of the PtCo-CdSe hybrid nanowires was performed using SQUID magnetometry. Hysteresis loops are presented for the PtCo-CdSe hybrid nanowires for both 300 and $4.2 \mathrm{~K}$ in Fig. 4 (22). The lower inset displays temperature-dependent magnetization measurements done in both zero-field cooled and field-cooled modes. The structures exhibit superparamagnetic behavior with a blocking temperature of approximately $30 \mathrm{~K}$, which is typical of nanoscale PtCo particles (23). We thus see that, in this case, the magnetic properties of the PtCo alloys are preserved upon growth onto the CdSe nanowires.

Optical absorption and photoluminescent (PL) measurements of the Au-CdSe hybrid nanowires show significant broadening upon $\mathrm{Au}$ growth a concurrent quenching of the photoluminescence indicative of strong coupling between the semiconductor and metal tip. Fig. 4 presents the SEM images (using the STEM detector) of the CdSe nanowires before (4A) and after $\mathrm{Au}$ growth (4B). The PL and absorption spectra of the same samples shown in A and B were taken and shown in C and D, respectively.

Electrical measurements of single nanostructures remain a challenging task. The electrical properties of a single semiconductor nanostructure are influenced by various parameters; among these parameters is the interface between the semiconductor nanostructure and the metal electrodes. This interface plays a significant role of determining the overall properties. We have conducted systematic measurements to study the effect of the interface by comparing the electrical response of CdSe nanorods with and without the Au tips (24). The AuCdSe hybrid nanorods provide a direct two metalsemiconductor interfaces with no surfactant in the middle.

Fig. 5 shows the TEM images CdSe nanorods with (Fig. 5A) and without Au tips (Fig. 5B) and the SEM images of the two $\mathrm{Au}$ electrodes that were fabricated as described in the experimental section (Fig. 5C). The absorption spectra of the CdSe sample before and after the growth are shown in Fig. 5D. The broadening of the absorption spectrum of the CdSe-Au sample was discussed earlier in this manuscript.

Measurement of the electrical properties of single nanords is shown in Fig. 6. The room temperature resistance of various devices is summarized in Fig. 6A. The resistance values obtained for the pure CdSe (red trace) are ranged between $10^{11}$ and $10^{13}$, which is 6-orders of magnitude higher compared to the Au-CdSe (green trace) nanostructures. These results can be shown in the 
A

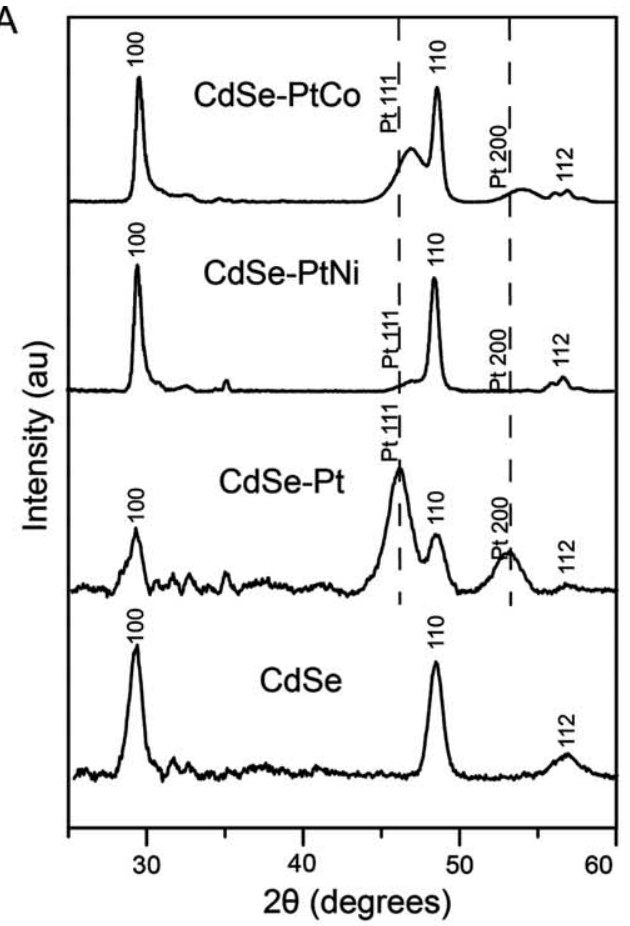

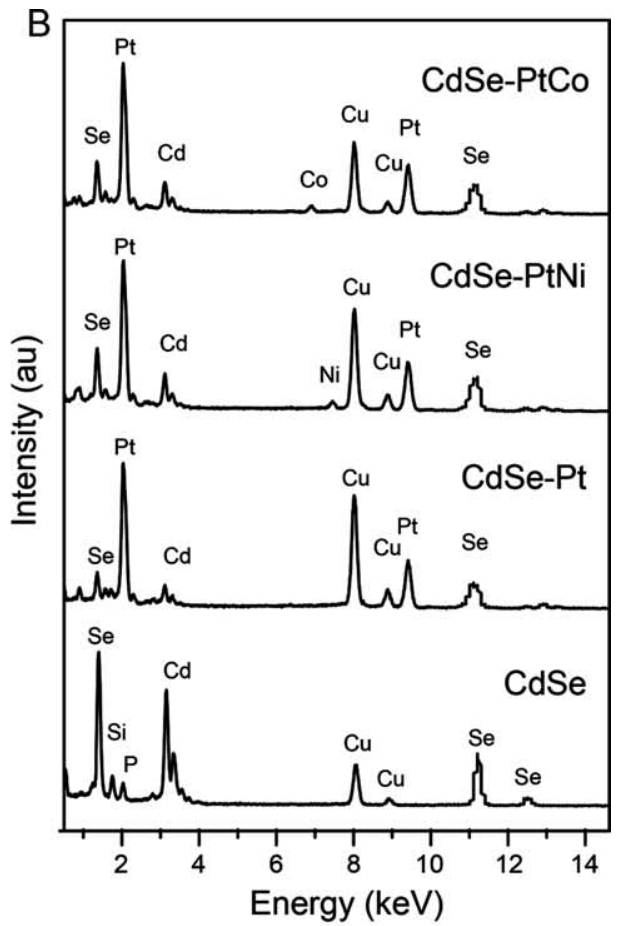

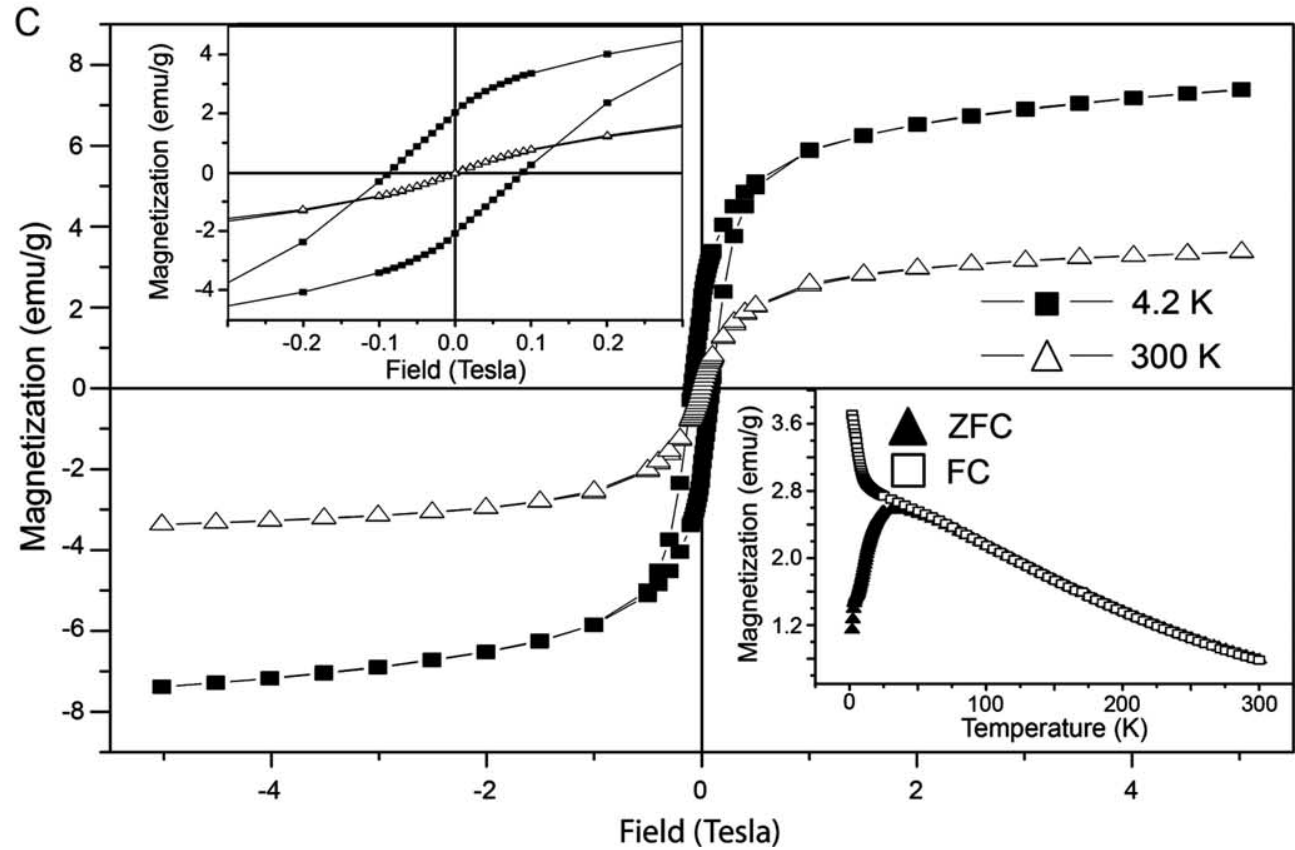

Characterization of metal and binary metal overgrowth on CdSe nanowires. (A) XRD data, (B) EDS data, (C) SQUID magnetometry results for CdSe-PtCo showing temperature-dependent magnetization measurements (magnified in upper inset). Lower inset shows traces of magnetization as a function of temperature in zero-field cooled (triangles) and field-cooled (squares) modes.

I-V curve (Fig. 6B), where the resistance the zero-bias is much higher for the CdSe nanorods compared to the AuCdSe hybrid structure. The improvement of the electrical conductivity by lowering the resistance at the interface opens a wide range of applications in electronics, energy, and others.

\section{Conclusion}

Hybrid nanostructures could have special interest in catalysis and energy application. We have shown the synthesis of hybrid nanostructures of different materials and various shapes. The electrical properties of individual CdSe nanorods with and without Au tips are shown. 

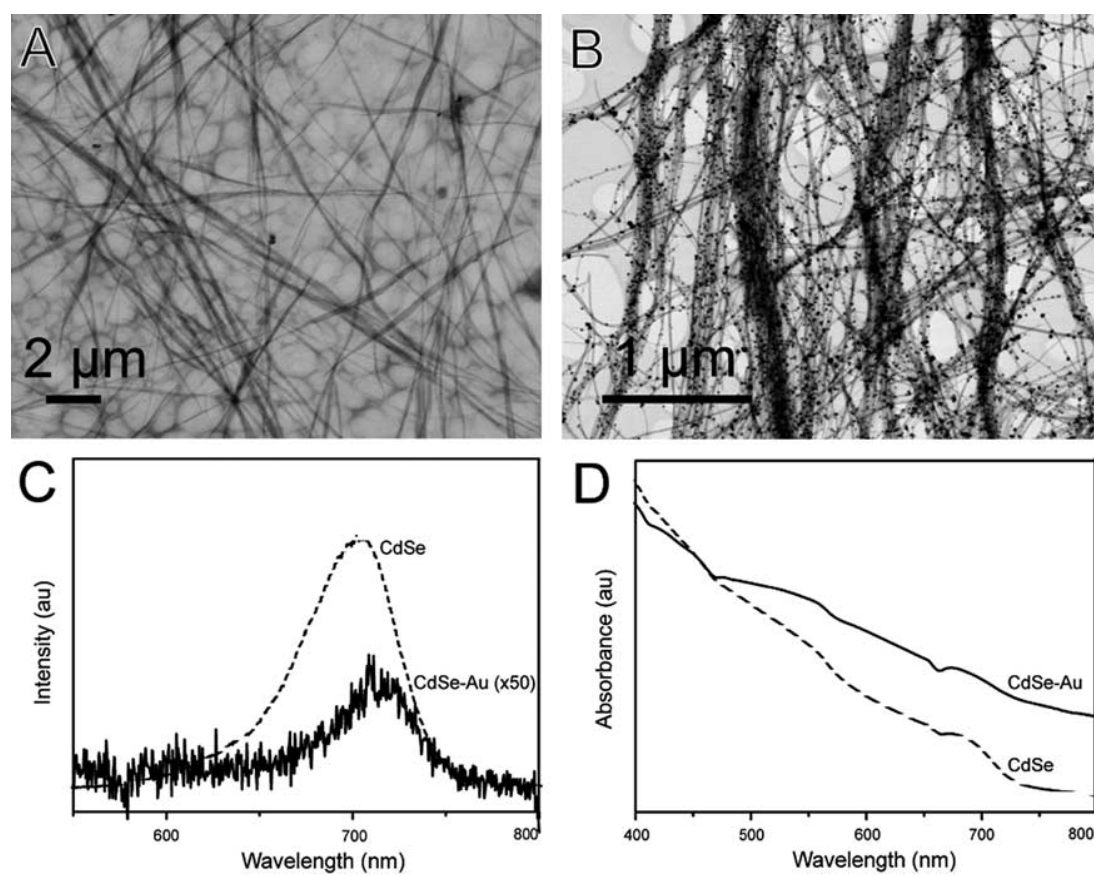

Growth of different metals and binary metals on CdSe nanowires. (A) CdSe-Au nanowires, (B) CdSe-Pt nanowires, (C) CdSe-PtNi nanowires, (D) CdSe-PtCo nanowires.
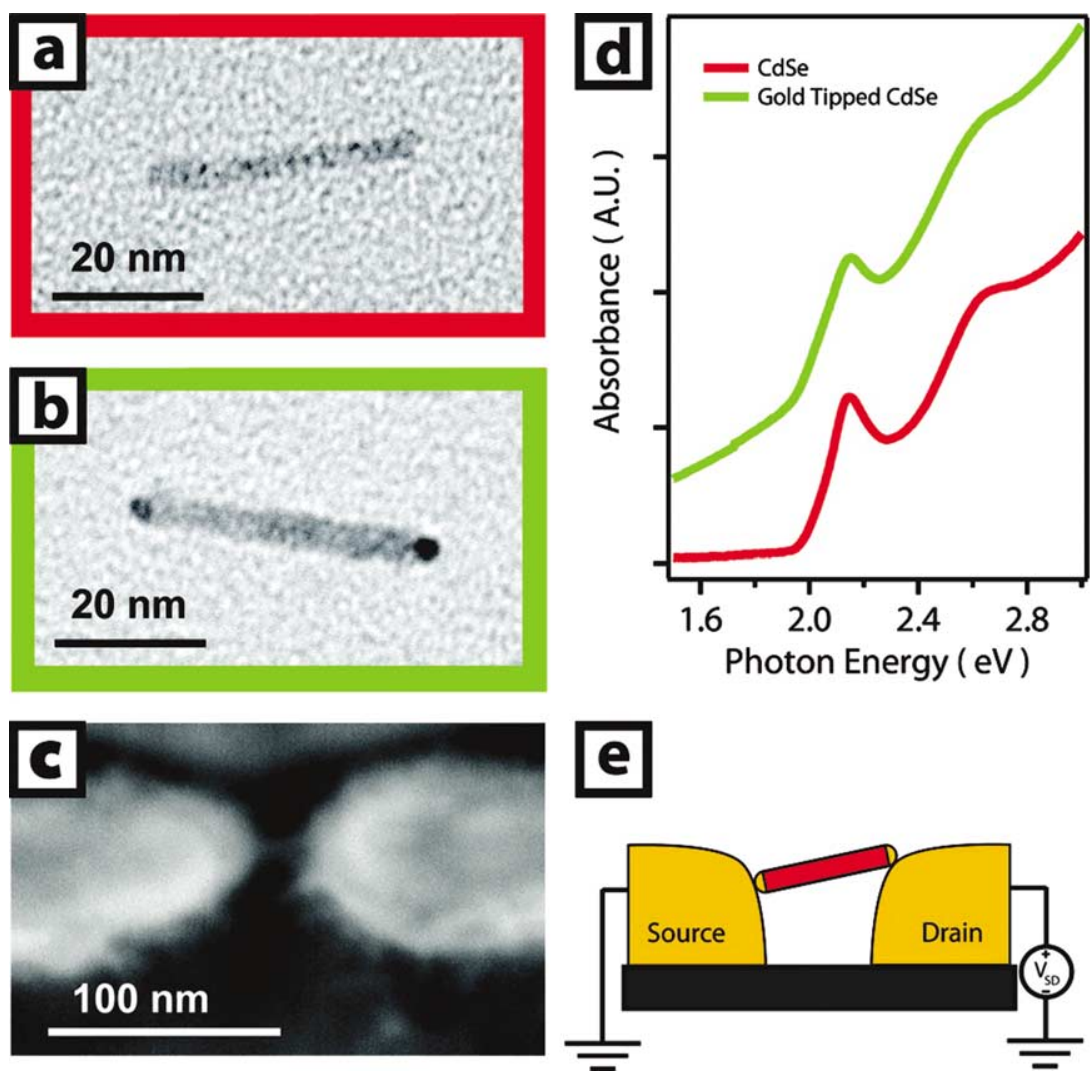

TEM image of CdSe nanocrystal before (A) and after (b) Au tip growth. SEM image (c) and schematic (e) of a single nanocrystal two-terminal device. After a silicon wafer test chip was submersed in a toluene-nanocrystal solution, the evaporating solvent orients individual nanocrystals across predefined Au electrodes fabricated via e-beam lithography. (d) Solution phase optical spectra indicate onset of first exciton absorption at $2 \mathrm{eV}$ for both CdSe (red) and Au-tipped CdSe heterostructure (green) samples. 

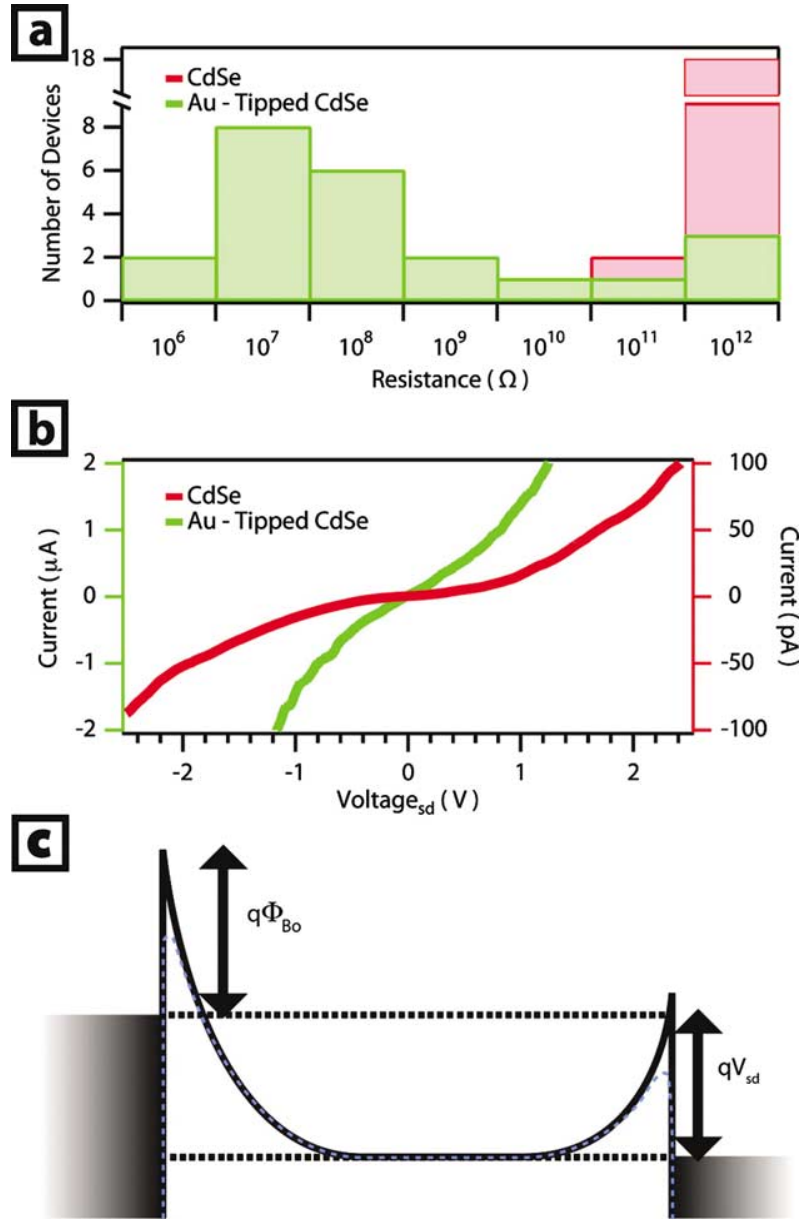

Fig. 6. (A) Histogram of room temperature device resistance near $0 \mathrm{~V}$ applied bias. (B) Representative two-probe $I-V$ trace of a CdSe device (red) and an Au-tipped CdSe device (green) at room temperature. Note the color-coded axes correspond to picoampere (red) and microampere (green) scales. (C) Simplified energy-band diagram of proposed barrier structure across a device under bias. The dashed blue line shows the barrier lowering due to the image potential - not drawn to scale.

\section{Conflict of interest and funding}

There is no conflict of interest in the present study for any of the authors.

\section{References}

1. Cozzoli PD, Pellegrino T, Manna L. Synthesis, properties and perspectives of hybrid nanocrystal structures. Chem Soc Rev 2006; 35: 1195-208.

2. Peng X, Manna L, Yang W, Wickham J, Scher E, Kadavanich A, et al. Shape control of CdSe nanocrystals. Nature 2000; 404: $59-61$.

3. Subramanian V, Wolf EE, Kamat PV. Green emission to probe photoinduced charging events in $\mathrm{ZnO}-\mathrm{Au}$ nanoparticles. Charge distribution and fermi-level equilibration. J Phys Chem B 2003; 107: 7479-85.

4. Pacholski C, Kornowski A, Weller H. Site-specific photodeposition of silver on $\mathrm{ZnO}$ nanorods. Angewandte Chemie Int Ed 2004; 43: 4774-7.

5. Casavola M, Grillo V, Carlino E, Giannini C, Gozzo F, Fernandez E, et al. Topologically controlled growth of magnetic-metal-functionalized semiconductor oxide nanorods. Nano Lett 2007; 7: 1386-95.

6. Kamat PV, Flumiani M, Dawson A. Metal-metal and metalsemiconductor composite nanoclusters. Coll Sur. A: Physicochem Eng Aspects 2002; 202: 269-79.

7. Yang J, Elim HI, Zhang Q, Lee JY, Ji W. Rational synthesis, selfassembly, and optical properties of PbS-Au heterogeneous nanostructures via preferential deposition. J Am Chem Soc 2006; 128: 11921-6.

8. Talapin DV, Yu H, Shevchenko EV, Lobo A, Murray CB. Synthesis of colloidal $\mathrm{PbSe} / \mathrm{PbS}$ core-shell nanowires and $\mathrm{PbS} /$ Au nanowire-nanocrystal heterostructures. J Phys Chem C 2007; 111: 14049-54.

9. Ahrenstorf K, Albrecht O, Heller H, Kornowski A, Görlitz D, Weller H. Colloidal synthesis of NixPt1-X nanoparticles with tuneable composition and size. Small 2007; 3: 271-4.

10. Kuykendall T, Aloni S, Jen-La Plante I, Mokari T. Growth of GaN@InGaN core-shell and Au-GaN hybrid nanostructures for energy applications. Int J Photoenergy 2009; Article No. 767951 .

11. Jacob T, Goddard WA. Adsorption of atomic $\mathrm{H}$ and $\mathrm{O}$ on the (111) surface of $\mathrm{Pt}_{3} \mathrm{Ni}$ alloys. J Phys Chem B 2004; 108: 8311-23.

12. Park KW, Choi JH, Kwon BK, Lee SA, Sung Y, Ha HY, et al. Chemical and electronic effects of $\mathrm{Ni}$ in $\mathrm{Pt} / \mathrm{Ni}$ and $\mathrm{Pt} / \mathrm{Ru} / \mathrm{Ni}$ alloy nanoparticles in methanol electrooxidation. J Phys Chem B 2002; 106: 1869-77.

13. Trentler TJ, Hickman KM, Goel SC, Viano AM, Gibbons PC, Buhro WE. Solution-liquid-solid growth of crystalline III-V semiconductors: an analogy to vapor-liquid-solid growth. Science 1995; 270: 1791-4.

14. Ouyang L, Maher KN, Yu CL, McCarty J, Park H. Catalystassisted solution-liquid-solid synthesis of $\mathrm{CdS} / \mathrm{CdSe}$ nanorod heterostructures. J Am Chem Soc 2007; 129: 133-8.

15. Khan OFZ, O'Brien P. Synthesis, characterization, $113 \mathrm{Cd}$ NMR and decomposition of some cadmium thiolate. Polyhedron 1991; 10: 325-32.

16. Habas SE, Yang P, Mokari T. Selective growth of metal and binary metal tips on CdS nanorods. J Am Chem Soc 2008; 130: 3294-5.

17. Mokari T, Rothenberg E, Popov I, Costi R, Banin U. Selective growth of metal tips onto semiconductor quantum rods and tetrapods. Science 2004; 304: 1787-90.

18. Saunders AE, Popov I, Banin U. Synthesis of hybrid CdS-Au colloidal nanostructures. J Phys Chem B 2006; 110: 25421-9.

19. Mokari T, Sztrum CG, Salant A, Rabani E, Banin U. Formation of asymmetric one-sided metal-tipped semiconductor nanocrystal dots and rods. Nat Mat 2005; 4: 855-63.

20. Jen-La Plante I, Habas S, Yuhas B, Gargas D, Mokari T. Interfacing metallic nanoparticles with semiconductor nanowires. Chem Mat 2009; 21: 3662-67.

21. Shevchenko EV, Talapin DV, Rogach A, Kornowski L, Haase $\mathrm{M}$, Weller H. Colloidal synthesis and self-assembly of CoPt3 nanocrystals. J Am Chem Soc 2002; 124: 11480-85.

22. Yuhas BD, Habas SE, Fakra S, Mokari T. Probing compositional variation within hybrid nanostructures. ACS Nano 2009; 3: $3369-76$. 
23. Wiekhorst F, Shevchenko E, Weller H, Kötzler J. Anisotropic superparamagnetism of monodispersive cobalt-platinum nanocrystals. Phys Rev B 2003; 67: 224416/1-224416/11.

24. Sheldon M, Trudeau P, Mokari T, Wang L, Alivisatos AP. Enhanced semiconductor nanocrystal conductance via solution grown contacts. Nano Lett 2009; 9: 3676-82.
*Taleb Mokari

Department of Chemistry and

Ilse Katz Institute for Nanoscale Science and Technology

Ben-Gurion University of the Negev

Beer Sheva, Israel

Email: mokari@bgu.ac.il 